\title{
Enhanced and Controllable Multilayer SERS Substrate with Composite Layer
}

\author{
Chunzhen Fan ${ }^{1, a^{*}}$ \\ ${ }^{1}$ School of Physical Science and Engineering, Zhengzhou University, Zhengzhou 450052, China \\ chunzhen@zzu.edu.cn
}

Keywords: Composites layer; SERS substrate; Local plasmon resonant; Controllable

\begin{abstract}
We numerically investigate the optical properties of the multilayer SERS structures. It includes the substrate layer, the adhesion layer and the composite layer which is consisting of the metallic nanoparticles embedding in the host metallic or dielectric layer. Under the quasi-static approximation, the effective dielectric constant of the composite layer is explored. Its enhanced electric field and the wave prorogating properties are fully explored with the aid of the Fresnel's equations. Our numerical results illustrate that the volume fraction of the metallic nanoparticles and the dielectric properties of the host medium can greatly affect the optical propagating properties (absorbance and the reflectance). Moreover, enhanced local electromagnetic field can be obtained with the metallic (gold) host medium other than the dielectric (TiO2). Thus, our multilayer SERS substrate with excellent controllable enhanced local electric filed endorsed us another degree to engineer the optical transmission. It is a promising candidate in the SERS biosensors applications.
\end{abstract}

\section{Introduction}

Surface-enhanced Raman spectroscopy (SERS) discovered in 1974 has emerged as a promising sensing tool in complex biological system [1-4]. The sensing principle relies on the shift of the plasmonic resonance caused by the surrounding dielectric environmental [5]. Surface plasmon indicates a resonant electromagnetic wave, where oscillating electromagnetic field incident on the nanoparticle, the conduction electrons associated with the metal begin to oscillate at the same frequency. This resonance effect accompanies with the locally enhanced electromagnetic fields occurs around the corner or surface of metal plasmonic active nanostructures that are smaller than the wavelength of the incident radiation. Due to their small size effect and tunable surface plasmonic resonance, metallic nanoparticles yield a wide range of applications in chemical and biological sensors, plasmonic system and so on [6].

SERS substrate is the keystone to apply its sensing detection ability. Resort to the help of metallic nanostructures, SERS substrate can be fabricated with the incorporation of nanoparticles, nanofilm or nanoarrays. It can be modulated with molecular recognition motifs to render diagnostic tools for optical imaging and therapeutic studies [7-10]. Recently, a novel hybrid spectroscopic technique combining surface plasmon resonance with SERS is proposed, which can complement each other to provide rapid and quantitative detection of unknown analytes binding to a gold substrate [11]. Incorporating the surface plasmon resonance of metallic nanostructures, we study the multilayer SERS substrate, the sensing detection of the molecules setting right above the composite metal layer. Chromium adhesion layer is commonly used as the dielectric substrate materials. Resort to the effective medium theory, the effective dielectric constant of the composite layer is investigated. By using the transfer matrix method, we numerically explore the enhanced electric field and controllable propogation properties in the multilayer structure. Controlling over the plasmoic SERS effect is discussed with the composite layer.

\section{Model of Multilayer SERS Substrate}

Our proposed multilayer structure consists of the glass substrate layer, the adhesion layer and the 
composite layer, which is shown in Figure 1. For the composites layer, the metallic nanoparticles (gold or silver) suspend in the host medium. To apply the SERS detection with our multilayer structure, the chemical bond attaching with the molecules is deposited on the top layer. In our numerical calculation thickness of the adhesion layer is taken as $3 \mathrm{~nm}$ and that of the composite layer is $44 \mathrm{~nm}$ [5]. For the composite layer the effective dielectric constant is calculated with the effective medium theory [10]. It describes the metallic nanoparticles embedded in the host medium under the quasi-static approximations. The effective linear dielectric constant of composite layer under present consideration $\varepsilon_{e}(\omega)$ can be obtained by the Maxwell-Garnett approximation.

$$
\frac{\varepsilon_{e}(\omega)-\varepsilon_{1}(\omega)}{\varepsilon_{e}(\omega)+2 \varepsilon_{1}(\omega)}=p \frac{\varepsilon_{2}-\varepsilon_{1}(\omega)}{\varepsilon_{2}+2 \varepsilon_{1}(\omega)}
$$

$\mathrm{p}$ is the volume fraction of the nanoparticles in the composite suspension. $\varepsilon_{1}(\omega)$ is the dielectric constant of the nanoparticles. $\varepsilon_{2}$ is the dielectric constant of host medium $\left(\varepsilon_{2}=2.5\right)$. The dielectric constant of the metallic nanoparticles $\varepsilon_{1}(\omega)$ observes the Drude form. $\omega=2 \pi c / \lambda$ is the discrete values of the frequency. The local enhanced field for the detection of the probe molecule is written as $E_{i}^{i+1}$. The intensity of the Raman scattered radiation is proportional to the square of the magnitude of any electromagnetic fields incident on the analyst. Resorting to the boundary conditions, namely, the continuity of the tangential magnetic field and the normal component of the electric excitation, the transfer equations can be listed out in the multilayer system based on the transfer matrix method. In the $i$ th layer, the incoming $E_{i}^{i+1^{+}}$and outgoing electric filed $E_{i}^{i+1^{-}}$through the neighboring $(i+1)$ th layer can be written as the following equation

$$
\left(\begin{array}{l}
E_{i}^{i+1^{+}} \\
E_{i}^{i+1^{-}}
\end{array}\right)=\frac{1}{t_{i}^{i+1}}\left(\begin{array}{cc}
e^{i \delta_{i}} & r_{i}^{i+1} e^{i \delta_{i}} \\
r_{i}^{i+1} e^{-i \delta_{i}} & e^{-i \delta_{i}}
\end{array}\right)
$$

where $r_{i}^{i+1}=\frac{\varepsilon_{i+1} k_{i}-k_{i+1} \varepsilon_{i}}{\varepsilon_{i+1} k_{i}+k_{i+1} \varepsilon_{i}}, t_{i}^{i+1}=\frac{2 \varepsilon_{i+1} k_{i}}{\varepsilon_{i+1} k_{i}+k_{i+1} \varepsilon_{i}}, r_{i}^{i+1}$ and $t_{i}^{i+1}$ are the Fresnel's reflection coefficient on the interface separating two mediums of relative permittivity $\varepsilon_{i}$ and $\varepsilon_{i+1}$ respectively. $k_{i}=k_{0} \sqrt{\varepsilon_{i}-\sin \theta^{2}}, k_{0}=2 \pi / \lambda, \lambda$ is the incident wavelength. $\delta_{i}=2 \pi n_{i} d_{i} \cos \theta / k_{i}, n_{i}$ is the refraction index and $d_{i}$ is the thickness of the $i$ th layer. The illumination is made by a $\mathrm{p}$ polarized plane wave. $\theta$ is the angle between the illumination wave vector, and the normal to the layer vector. The wave vector differs from the relative permittivity of the medium of detection and therefore it could influence the efficiency of the multilayer SERS biosensor.

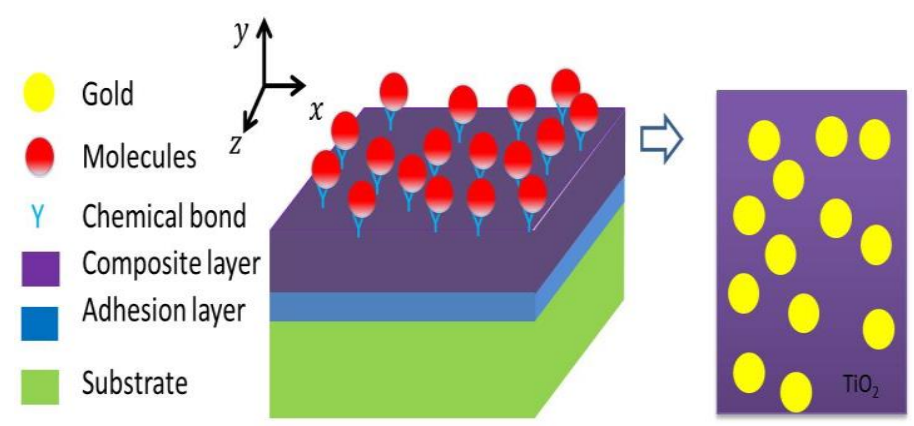

Figure 1. Schematic multilayer SERS substrate, it composes of the substrate layer, the adhesions layer, the composite layer and the detection biological molecules. The composite layer consists of the gold nanoparticles suspending in the host medium $\mathrm{TiO}_{2}$. 

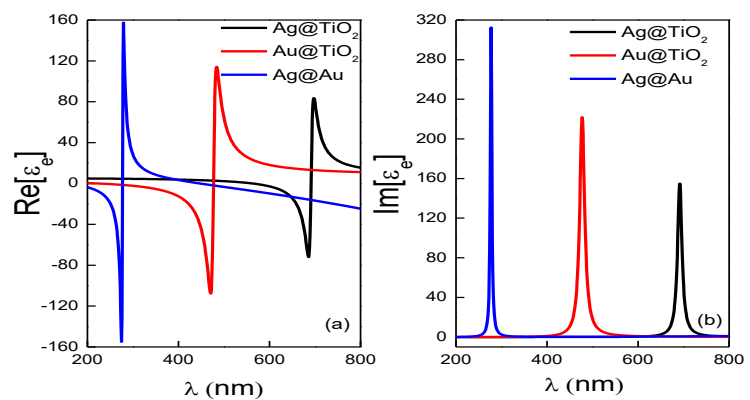

Figure 2. Real and imaginary part of the effect dielectric constant of three different composite layers (Ag@ $\left.\mathrm{TiO}_{2}, \mathrm{Au} @ \mathrm{TiO}_{2}, \mathrm{Ag} @ \mathrm{Au}\right)$. The volume fraction of the metallic nanoparticles p is taken as 0.45 .

\section{Results and Discussion}

In this section, we present our numerical results (figures 2-4) of multilayer SERS structure. Specifically, metallic nanoparticles suspend in dielectric /metallic medium are investigated. Figure 2 illustrates the real and imaginary part of the effective dielectric constant of three different composite layers (Ag@ $\mathrm{TiO}_{2}, \mathrm{Au} @ \mathrm{TiO}_{2}, \mathrm{Ag} @ \mathrm{Au}$ ) as a function of incident wavelength from $200 \mathrm{~nm}$ to $800 \mathrm{~nm}$. Here we take the volume fraction of the nanoparticles as 0.45 . The real and imaginary part of the effective dielectric constant of the composite layer can be greatly affected when the suspended nanoparticles silver or gold is included in dielectric medium. Moreover, the host medium has a significant effect on the effective permittivity. Enhanced absorption can be obtained when the metallic nanoparticles embedded in the host metallic medium, which was shown as blue curve in Figure 2(b).
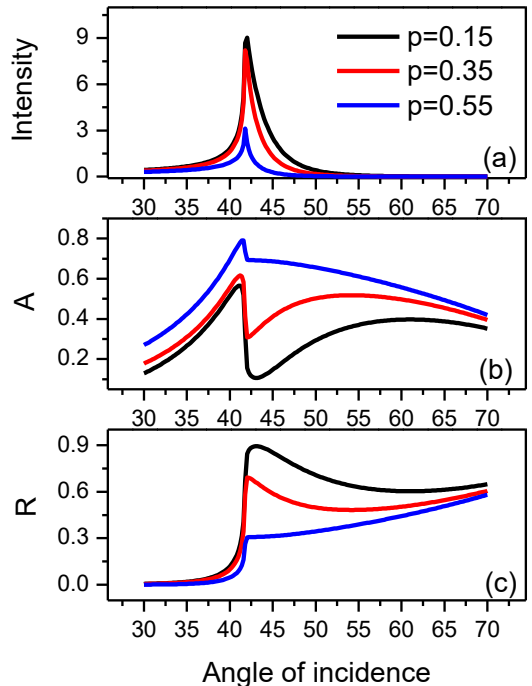
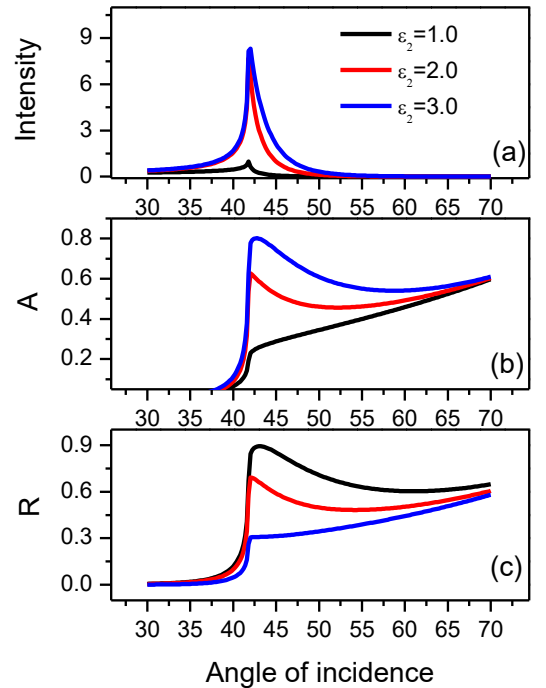
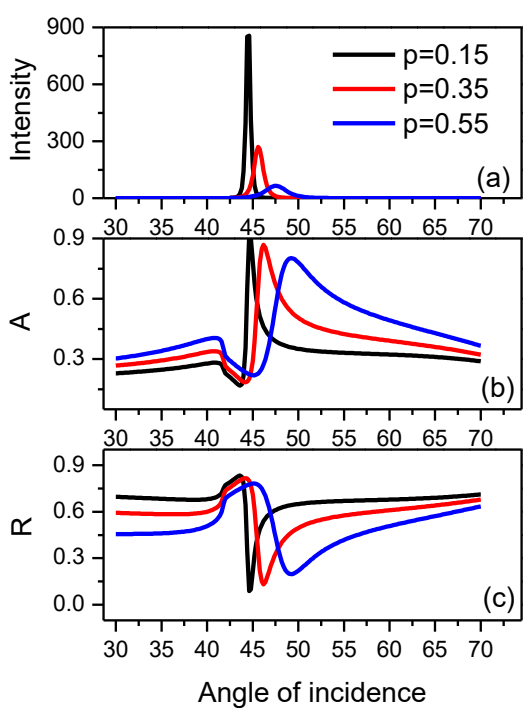

Figure 3. The local electric field, the absorbance and the reflectance of the multilayer SERS substrate with $\mathrm{Ag} @ \mathrm{TiO}_{2}$ composite layer are calculated as a function of the incident angle by tuning the volume fraction of the silver nanoparticles $(\mathrm{p}=0.15,0.35$, and 0.55$)$.

Figure 4. The local electric field, the absorbance and the reflectance of the multilayer SERS substrate with $\mathrm{Ag} @ \mathrm{TiO}_{2}$ are calculated with different dielectric constant of the host medium $\left(\varepsilon_{2}=1.0,2.0\right.$, and 3.0).

Figure 5. The local electric field, the absorbance and the reflectance of the multilayer SERS substrate with $\mathrm{Ag} @ \mathrm{Au}$ are calculated by tuning the volume fraction of the silver nanoparticles $(\mathrm{p}=0.15,0.35$, and 0.55$)$. 
Fig 3. examines the local electromagnetic field, absorption and reflection of the model multilayers SERS substrate by tuning the volume fraction of metallic Ag nanoparticles. In this case, we set the host medium is the dielectric $\mathrm{TiO}_{2}$ layer. The results illustrate that decreased $\mathrm{p}$ can induce larger electromagnetic field intensity. Moreover, the absorbance can be greatly enhanced with a larger $\mathrm{p}$. Thus, our numerical results show that the composite layer plays an important role in the multilayer SERS substrate to achieve the enlarged local electromagnetic field. The comparison of the SERS enhancement and propagating properties for different host medium is shown in Fig. 4. Here we set the host medium $\varepsilon_{2}$ as 1.0, 2.0 and 3.0 respectively. Enhanced intensity can be obtained when the host medium $\varepsilon_{2}$ got increased. The absorbance and reflectance can also be tuned with varied $\varepsilon_{2}$

Now we are in a position to consider the metallic host medium effect on the multilayer SERS structure. It is clearly observed that significantly enhanced local magnetic field can be obtained in the bimetallic composite layer. It is two orders of magnitude higher than that in the dielectric medium. More precisely, local electromagnetic filed is close to 900 when the $\mathrm{p}$ is taken as 0.15 . It was caused by the hybridization effect of the local plasmonic resonant of the nanoparticles and the propagating effect of the metallic layer. Moreover, the peak of absorbance and reflectance move to the large incident angle region when the volume fraction of the metallic nanoparticles $\mathrm{p}$ is approaching 0.45. Our results reveal that enhanced and tunable SERS intensity can be achieved by properly selection of the volume fraction and host medium in the composite layer.

\section{Summary}

In summary, we report a novel multilayer SERS substrate, which is consisting of the metallic silver or gold nanoparticles in the host dielectric or metallic medium. The local electromagnetic field, the reflectance and transmittance of our novel substrates are calculated with the effective medium theory. With the change of the volume fraction metallic nanoparticles or the dielectric host medium, our SERS probes are based on the enhancement electromagnetic field caused by the proximity of Raman active signature molecules to the surface of metal nanoparticles. This work demonstrates that, when the nanostructures are properly designed, enhanced and tunable Raman signal can be obtained.

\section{Acknowledgements}

This work is supported by the Key science and technology research project of Henan Province (162102210164), by the Foundation of Henan Educational Committee (17A140002).

\section{References}

[1] N.M. S. Sirimuthu, C. D. Syme, J. M. Cooper, Anal. Chem. 2010, 82, 7369-7373.

[2] B. Yan, S. V. Boriskina, and B. M. Reinhard ,J. Phys. Chem. C 2011, 115, 24437-24453

[3] Z. Skeete, H. Cheng, E. Crew, L. Lin, W. Zhao, P. Joseph, S. Y. Shan, H. Cronk, J. Luo, Y. Li, Q. Zhang, and C. J. Zhong, Appl. Mater. Interfaces 2014, 6, 21752-21768

[4] R.V. Sondergaard, N. M. Christensen, J. R. Henriksen, E. K. Pramod Kumar, K. Almdal, and T. L. Andresen Chem. Rev. 2015, 115, 8344-8378

[5] [D. Barchiesi, T. Grosges, F. Colas and M. L. de la Chapelle, J. Opt. 17 (2015) 114009.

[6] C.F. Bohren and D.R. Huffman Absorption and Scattering of Light by Small Particles (Wiley-Inter science Publication, John Wiley \& Sons) 1998.

[7] S. A. Meyer, B. Auguié, E. C. Le Ru, and Pablo G. Etchegoin, J. Phys. Chem. A, 2012, 116 (3), 1000-1007

[8] J. Salvi Dominique Barchiesi, Appl. Phys. A (2014) 115:245-255 
[9] D. Barchiesi , N. Lidgi-Guigui, M. L.de la Chapelle, Opt. Commun. 285 (2012) 1619-1623 [10] J. P. Huang and K.W. Yu 2006 Phys. Rep. 4313 